\title{
Construção de uma carta celeste: Um recurso didático para o ensino de Astronomia nas aulas de Física
}

\author{
Construction of a sky chart: A teaching resource for astronomy teaching in Physics classes \\ Artur Justiniano*1, Rafael Botelho ${ }^{2}$ \\ ${ }^{1}$ Universidade Federal de Alfenas, Alfenas, MG, Brasil \\ ${ }^{2}$ Instituto Nacional de Pesquisas Espaciais, São José dos Campos, SP, Brasil
}

Recebido em 10 de junho de 2016. Aceito em 20 de julho de 2016

\begin{abstract}
Uma carta celeste é um mapa do céu utilizado para identificar e localizar os objetos astronômicos. Até o início do século XX elas eram essenciais em diversas atividades humanas como a navegação e o planejamento econômico. Hoje em dia, há diversos softwares e aplicativos para celular capazes de confecciona-las em tempo real. Neste trabalho apresentamos um método para construir uma carta celeste que pode ser utilizado como um recurso didático para introduzir conceitos de astronomia nas aulas de física. O trabalho está dividido em três partes. Na primeira vamos apresentar os dois sistemas de coordenadas celeste, o equatorial e o horizontal. Na segunda as definições de tempo que serão utilizadas. $\mathrm{Na}$ terceira apresentamos como calcular as coordenadas horizontais dos astros e construir a carta celeste. Para finalizar apresentamos um comparação dos nossos resultados com os da literatura. Nas considerações finais há uma sugestão de como abordar esse conteúdo na sala de aula.
\end{abstract}

Palavras-chave: astronomia, carta celeste, ensino de astronomia

A star chart is a map of the sky used to identify and locate astronomical objects. By the early twentieth century they were essential in various human activities such as human navigation and economic planning. Today, there are various software and mobile applications able to build them in real time. In this work, we present a method to build a sky chart that can be used as a teaching tool to introduce astronomy content in physics classes. The work is divided into three parts. At first, we present the two celestial coordinate systems, equatorial and horizontal. In the second, the time settings. In the third, we show how to calculate the horizontal coordinates of the stars and build a sky chart. Finally, we present a comparison of our results with the literature. In the conclusion, a suggestion of how to approach this this subject in the classroom.

Keywords: astronomy, star chart, astronomy education

\section{Introdução}

Uma carta celeste é um mapa do céu. Esse mapa é utilizado para identificar e localizar os objetos astronômicos. Principalmente planetas, constelações e estrelas.

A confecção dessas cartas remonta as primeiras civilizações. O registro mais antigo, data de aproximadamente 32500 anos [1]. Trata-se de uma escultura em um bloco de marfim encontrada em 1979

\footnotetext{
*Endereço de correspondência: arturjustiniano@gmail.com
}

em uma caverna na Alemanha e que se assemelha à constelação de Órion.

Até o início do século XX as cartas celestes eram essenciais para a navegação marítima, na confecção do calendário, na identificação das estações do ano e no planejamento da atividade econômica. Conhecer a posição e o movimento dos corpos celeste era a forma que se tinha para se localizar, medir a passagem do tempo e determinar os períodos de plantar e colher [2]. Com o avanço científico e o advento de 
novas tecnologias para medir a passagem do tempo e se localizar, as cartas celeste passaram a ser utilizadas quase que exclusivamente para a divulgação, o ensino e a pesquisa em astronomia.

A popularização do uso das cartas celeste pode ser observada nos diversos softwares, tanto para computadores quanto para smartphones, que produzem em tempo real um mapa do céu para qualquer local do planeta. O celestia, o stellarium, o cartes do ciel, o skeye e o skyMap são exemplos de alguns dentre muitos dos softwares disponíveis.

É importante esclarecer que existem algumas diferenças entre os softwares para computadores e para os smartphones. Nos PCs o usuário precisa informar para qual localidade pretende construir a carta celeste e para qual data. Essas informações são essenciais para que o software faça a simulação da carta celeste em uma determinada plataforma gráfica. Já alguns aplicativos para os smartphones permitem ao usuário visualizar o mapa do céu para a posição que o aparelho estiver direcionado. Para isto o aparelho utiliza o GPS e sensores de movimento, o acelerômetro e o giroscópio, para se localizar no tempo e no espaço. Com essas informações o software cria uma simulação da esfera celeste para o local onde está o usuário.

Aparentemente parece um processo complicado a construção de uma carta celeste. Pode-se imaginar que são cálculos complicados, extensos e que podem demandar horas de trabalho se forem feitos sem a utilização de um programa de computador. Nesse artigo vamos tentar derrubar esse mito. Vamos mostrar como construir uma carta celeste com alguns conhecimentos de astronomia de posição e uma planilha de cálculo.

Inicialmente o método foi desenvolvido para responder a solicitação dos alunos que queiram saber como são feitos os mapas do céu nos aplicativos dos smartphones. Posteriormente, observamos que ele é um recurso didático que pode ser utilizado para trabalhar conceitos de Astronomia nas aulas de física, geografia e matemática por se entender que o tema apresenta capacidade motivadora e é potencialmente capaz de permitir o estabelecimento de conexões com diferentes áreas do conhecimento.

A Fig.1 é um esquema dos passos necessários para a construção da carta. Observem que para construí-la são necessárias três informações de entrada - dados do astro, momento da observação e o local da observação - que vão produzir duas coordenadas na saída, azimute e altura. São essas coordenadas, para cada uma das estrelas, que são utilizadas para construir o mapa do céu. O primeiro passo é calcular o Tempo Sideral Local (TSL), em seguida o ângulo horário $(\mathrm{AH})$ de cada uma das estrelas. Com essa informação e conhecendo as coordenadas equatoriais das estrelas e as coordenadas geográficas do observador, podemos calcular as coordenadas horizontais das estrelas e construir a carta celeste.

\section{Os sistema de Coordenadas Celeste.}

Além do sistema de coordenadas geográficas que utilizada duas coordenadas, latitude e longitude, para localizar um objeto na superfície da Terra, vamos precisar de dois sistemas de coordenadas celeste [3]

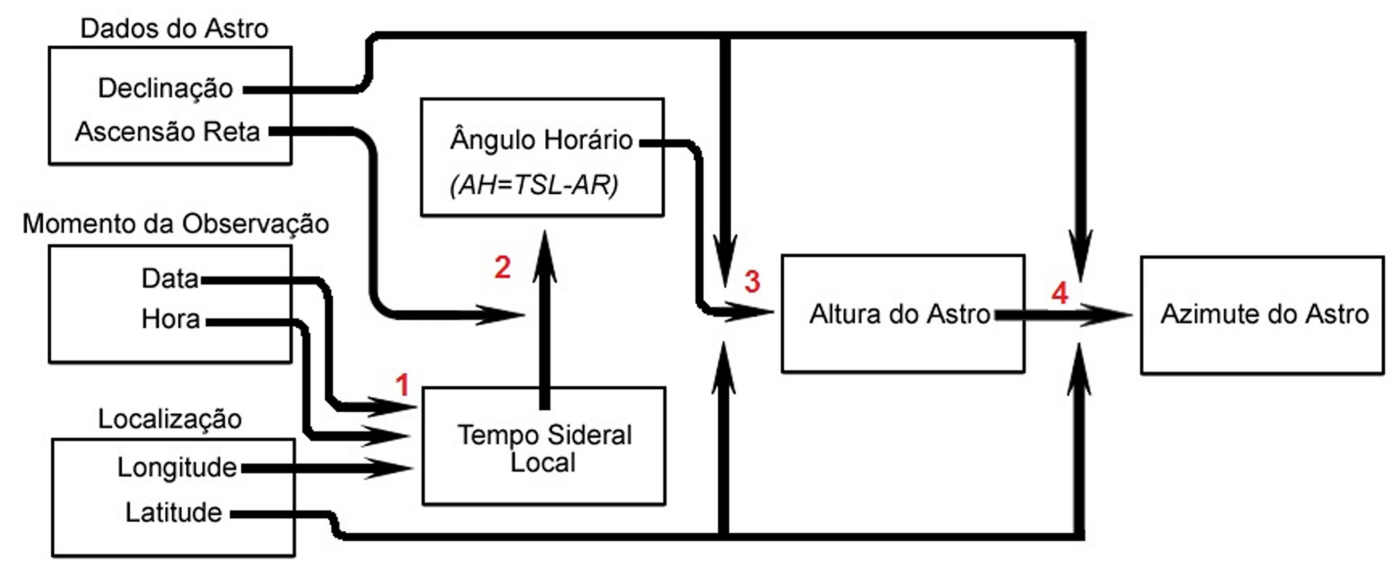

Figura 1: Diagrama com os passos necessários para calcular as coordenadas horizontais das estrelas. Os números correspondem as etapas que devem ser seguidas para calcular essas coordenadas e construir a carta celeste. 
para construir a carta. O sistema de coordenadas horizontal e o sistema de coordenadas equatorial.

O sistema horizontal possui duas coordenadas: o azimute(Az) e a altura(h). O azimute é um ângulo, medido sobre o horizonte, com origem no norte e que cresce na direção do leste, cuja extremidade é o meridiano do astro. Definido dessa forma o azimute varia de $0^{0} \leqslant \mathrm{Az}<360^{0}$. Já a altura é um ângulo, medido sobre o meridiano do astro, com origem no horizonte e extremidade no astro. Definida dessa forma a altura varia de $0^{0} \leqslant \mathrm{~h} \leqslant 90^{0}$ para os astros que estão acima do horizonte.

A Fig.2 é uma representação desse sistema de coordenadas. As setas azuis correspondem às duas coordenadas do sistema horizontal, Az e h. Pelo fato dos valores das coordenadas deste sistema dependerem das coordenadas geográficas do observador, um astro tem valores diferentes de $\mathrm{Az}$ e $\mathrm{h}$ para dois observadores em locais diferentes.

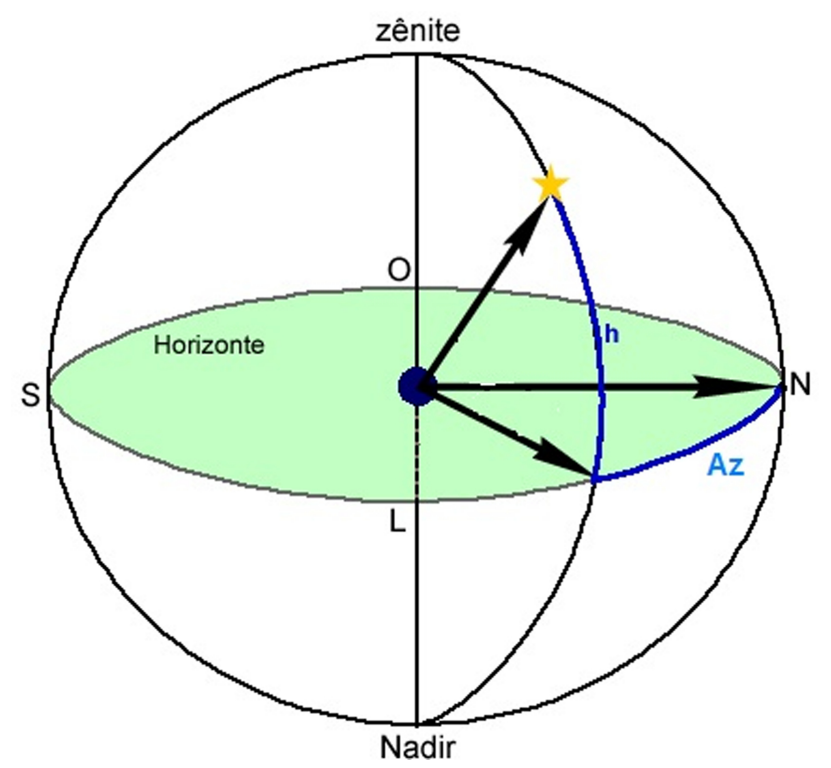

Figura 2: Sistema de coordenadas horizontal. As coordenadas da estrela são o azimute $(A z)$ e a altura(h). Zênite e nadir são os pólos desse sistema.

Uma forma conveniente de descrever a localização dos astros é assumir que eles estão na superfície de uma grande esfera cujo no centro está a Terra. Essa esfera, imaginária, é chamada de esfera celeste, e a intercessão do plano do equador terrestre com a esfera celeste é chamado de equador celeste. As projeções dos pólos terrestres na esfera celeste definem os pólos celeste Norte e Sul. A Fig. 3 mostra a esfera celeste e a trajetória aparente do Sol ao redor da Terra durante o ano. Essa trajetória é chamada de eclíptica. Notem que o plano da eclíptica está inclinado em relação ao equador celeste. $\mathrm{O}$ ponto em que a eclíptica intercepta o equador celeste é definido como ponto de áries ou ponto gama $(\gamma)$. Quando o Sol está nesse ponto temos o início do outono no hemisfério sul e a primavera no hemisfério norte.

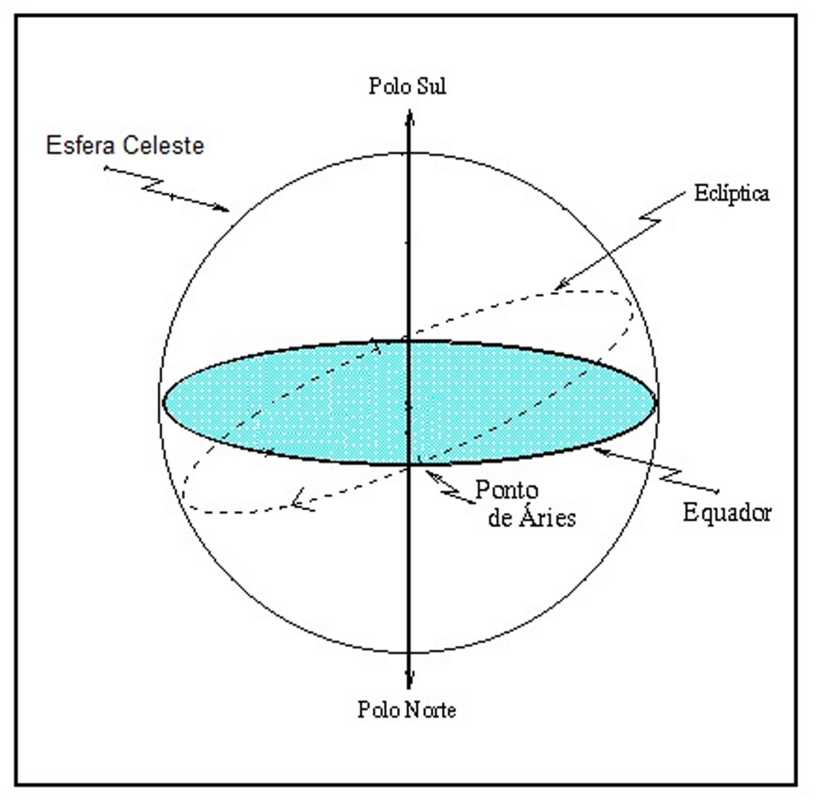

Figura 3: A esfera celeste. A linha tracejada, inclinada em relação ao equador celeste é a trajetória aparente do sol ao longo do ano, a eclíptica. O ponto de áries marca o local que o Sol cruza o equador celeste vindo do hemisfério Sul. Modificado da Ref. [3].

O sistema de coordenadas equatorial (Fig. 4) utiliza o ponto gama como referência. A primeira coordenada desse sistema, a ascensão reta $(\alpha)$, é o ângulo entre o meridiano do ponto gama e o meridiano do astro, medido sobre o equador celeste. Por convenção a ascensão reta é medida em horas, minutos e segundos (ao invés de graus, minutos e segundos de arco). Definida dessa forma a ascensão reta varia de $0 \leqslant \alpha<24$ horas. A segunda coordenada, a declinação $(\delta)$, é o ângulo entre o astro e o equador celeste, medido sobre o meridiano do astro. Quando medido na direção do pólo norte celeste $\delta \geqslant$ 0 , caso contrário a declinação é negativa. Definida dessa forma a declinação varia de $-90^{0} \leqslant \delta \leqslant 90^{\circ}$.

Das definições dos dois sistemas de coordenadas celeste podemos observar que no sistema horizontal as coordenadas de uma estrela variam continuamente devido a rotação da Terra e são diferentes para observadores em diferentes localizações. Já no sistema equatorial, para evitar essa variação, as coordenadas do astro independem da posição do 
observador na superfície da Terra e variam muito lentamente com o tempo, o que acarreta pequenas correções apenas ao longo dos anos.

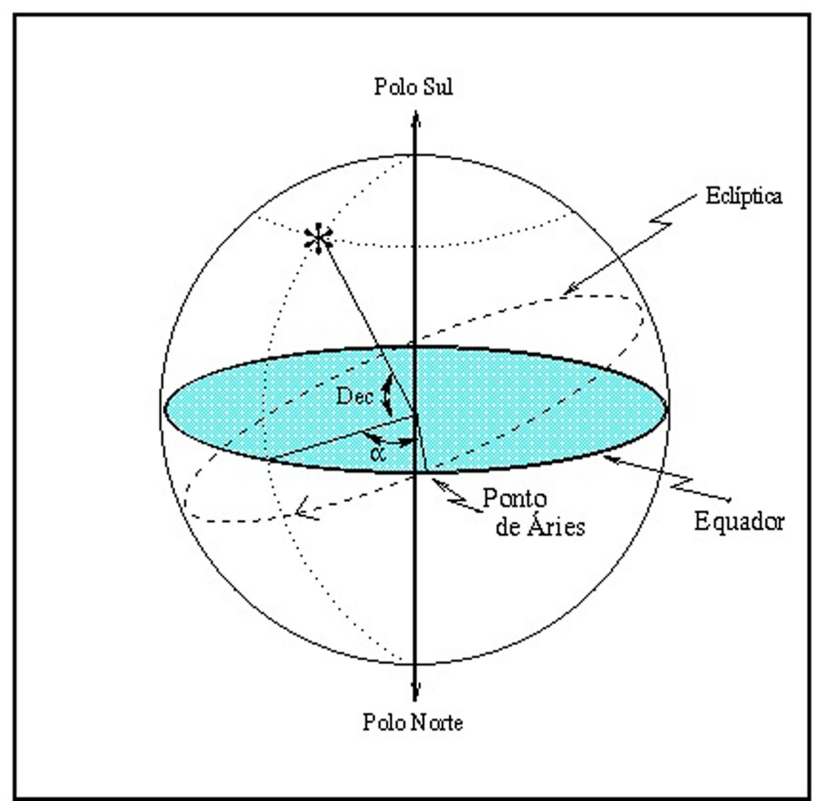

Figura 4: Sistema de coordenadas equatorial. As coordenadas da estrela são ascensão reta $(\alpha)$ e a declinação $\delta$. A linha traceja do pólo norte ou pólo sul é o meridiano da estrela e a linha tracejada inclinada em relação ao equador celeste é a eclíptica [3].

\section{A contagem do tempo.}

Nesta seção vamos explicar como converter as coordenadas do momento da observação(data e hora) em dia juliano (JD) e em seguida, conhecendo a longitude $(\phi)$ do local para qual se deseja construir a carta celeste, calcular ao Tempo Sideral Local (TSL). Explicações mais aprofundadas sobre a conversão da data em dia juliano e sobre o cálculo do TSL podem ser encontradas nas Refs.[4,5]

Em diversos cálculos astronômicos é necessário conhecer o número de dias transcorridos entre um evento e outro. Para facilitar esse cálculo foi criado um sistema onde os dias são contados de maneira consecutiva, sem interrupções em anos ou meses. Nesse sistema, uma data (ano, mês, dia, hora, minutos, segundos) é convertida para um número chamado de dia juliano (JD). Por definição, o dia juliano é o número de dias que se passaram desde o dia 1/1/4713 A.C às 12 horas em Greenwich.

Antes de apresentarmos como é feita a conversão de uma data do calendário gregoriano para dia juliano, são necessárias algumas explicações: a) Para essa conversão os meses são numerados de 1 (um) a 12 (doze). Sendo que 1 é o mês de janeiro e 12 o mês de dezembro.

b) Vamos utilizar a abreviação INT para designar a parte inteira de um número.

Conhecendo essas duas definições podemos agora apresentar como é feita a conversão da data para dia juliano. Vamos fazer isso dividindo os cálculos em partes.

A primeira parte é verificar se o mês (M) é janeiro ou fevereiro, ou seja, 1 ou 2. Se for, então a variável M é escrita assim: $\mathrm{M}=\mathrm{M}+12$ e a variável Ano é Ano $=$ Ano-1. Se o mês não for 1 ou 2 , então $\mathrm{M}=$ $\mathrm{M}$ e $\mathrm{Ano}=$ Ano.

A segunda parte é calcular $\mathrm{A} 1=\mathrm{INT}(\mathrm{Ano} / 100)$ e $\mathrm{B}=2-\mathrm{A} 1+\mathrm{INT}(\mathrm{A} 1 / 4)$. Se a data for anterior a $15 / 10 / 1582$ faça $\mathrm{A} 1=0$ e $\mathrm{B}=0 . \mathrm{A} 1$ e $\mathrm{B}$ são variáveis. A variável B será utilizada no cálculo de JD.

A terceira e última parte é o cálculo do dia Juliano [4].

$$
\begin{aligned}
& J D=B+I N T(365.25 * \text { Ano }-T) \\
& +I N T(30.60001 *(M+1)+D i a \\
& +\frac{\text { Hora }}{24}+1720944.5
\end{aligned}
$$

onde $\mathrm{T}=0$ se Ano $>0$, ou seja, para datas depois de Cristo e $\mathrm{T}=0.75$ para Ano $<0$, antes de Cristo.

A medida do tempo está relacionada ao movimento de rotação da Terra que provoca a rotação aparente da esfera celeste. Uma escala básica da medida do tempo é a definição do dia - tempo de uma rotação da Terra sobre seu eixo. Para medir essa rotação temos que usar um objeto celeste como referência. De acordo com o objeto tomado como referência temos dois tipos de tempo: o tempo solar, que toma como objeto de referência o Sol, e o tempo sideral, que usa como objeto de referência o ponto gama [5]. Esse segundo será alvo de nosso estudo.

Para determinar o Tempo Sideral Local (TSL) é necessário conhecer qual é o Tempo Sideral em Greenwich (TSG). Para ajudar o leitor a entender o que é o tempo sideral vamos imaginar a seguinte situação: Imagine que você esteja em Greenwich e que nesse exato momento o ponto gama esteja cruzando seu meridiano. Nesse momento o TSG será zero horas ou 24 horas. Para um observador na cidade de São Paulo que está a -3 horas de Greenwich o TSL será 21 horas. Ou seja, faltam 3 horas para o ponto gama cruzar o meridiano da cidade de São 
Paulo. Assim, para cada meridiano podemos estabelecer uma relação entre TSG com o TSL da seguinte forma [4]:

$$
T S L=T S G+\frac{\text { longitude }}{15}
$$

Agora vamos mostrar como determinar o TSG para uma determinada data. Primeiro é necessário calcular o dia juliano para a data que deseja construir a carta celeste (Eq.1) e a data juliana para a seguinte data 0/1/ano, que chamaremos de JD0. Em seguida calcule o número de dias (ndias) transcorridos desde o início do ano até o dia para qual a carta celeste está sendo construída da seguinte forma:

$$
\text { ndias }=\mathrm{JD}-\mathrm{JD} 0
$$

Agora é necessário calcular o século juliano (S), que por definição é o intervalo de tempo de 36525 dias.

$$
S=\frac{J D 0-2451545}{36525}
$$

Em seguida é necessário fazer os seguintes cálculos:

$$
\begin{aligned}
& T S S=6.6938 \times 3600+8640184.812866 \\
& \times S+0.093104 \times S^{2}-\left(\frac{6 \times 10^{-6} \times S^{3}}{3600}\right)
\end{aligned}
$$

e por fim,

$$
\begin{aligned}
& G S T=0.0657098 \times \text { dias } \\
& -(24-T S S+24 \times(\text { ano }-1900)) \\
& +1.00273790935 \times \text { Hora }
\end{aligned}
$$

Se

$$
G S T<0 G S T=G S T+24 .
$$

e se

$$
G S T>24 G S T=G S T-24 .
$$

\section{O ângulo Horário e a determinação das coordenadas horizontais das estrelas.}

O ângulo horário de um astro é uma medida de tempo [5]. Essa grandeza estabelece a distância angular entre o astro e o meridiano do observador, medida em horas. Por exemplo, no momento que uma estrela cruza o meridiano do observador o seu $\mathrm{AH}=0$ horas. Quando ela nasce à leste o $\mathrm{AH}=$ 18 horas e quando ela se põe à oeste o $\mathrm{AH}=6$ horas. Notem (Fig.5) que devido a forma como o ângulo horário é definido ele varia com o local e com o momento da observação, e esse momento é quantificado na forma de tempo sideral.

Assim, existe uma relação entre o ângulo horário o tempo sideral local e a ascensão reta do astro que é dada por [5]:

$$
\mathrm{AH}=\mathrm{TSL}-\alpha .
$$

Se $\mathrm{AH}<0 \mathrm{AH}=\mathrm{AH}+24$.

Como vimos, conhecendo o TSL para o momento que se deseja construir a carta celeste é possível calcular o $\mathrm{AH}$ das estrelas e assim determinar àquelas que estão acima do horizonte naquele TSL. Sabendo disso, e conhecendo as coordenadas equatoriais das estrelas e as coordenadas geográficas do local da observação, podemos calcular as coordenadas horizontais de cada uma delas e construir a carta celeste. As equações de transformação [4] são:

$$
\begin{gathered}
h=\operatorname{sen}^{-1}[\operatorname{sen}(\delta) \operatorname{sen}(\varphi) \\
+\cos (\delta) \cos (\varphi) \cos (A H)] \\
A z=\cos ^{-1}\left[\frac{\operatorname{sen}(\delta)-\operatorname{sen}(\varphi) \operatorname{sen}(A H)}{\cos (\varphi) \cos (A H)}\right]
\end{gathered}
$$

Se $\mathrm{AH}>0 \mathrm{Az}=360^{0}-\mathrm{Az}$.

\section{A carta Celeste}

Para construir a carta celeste nós selecionamos as estrelas mais brilhantes das doze constelações zodiacais e de mais 10 constelações. Utilizamos a Ref [6] para obter as coordenadas equatoriais das estrelas e montamos uma tabela (Apêndice). A partir dessa tabela nós calculamos para cada uma das estrelas as coordenadas horizontais ( $\mathrm{Az} \mathrm{e} \mathrm{h}$ ).

A Fig.6 é a carta celeste para o dia 6/6/2016 às 20:00 horas nas coordenadas geográficas, latitude $=$ 


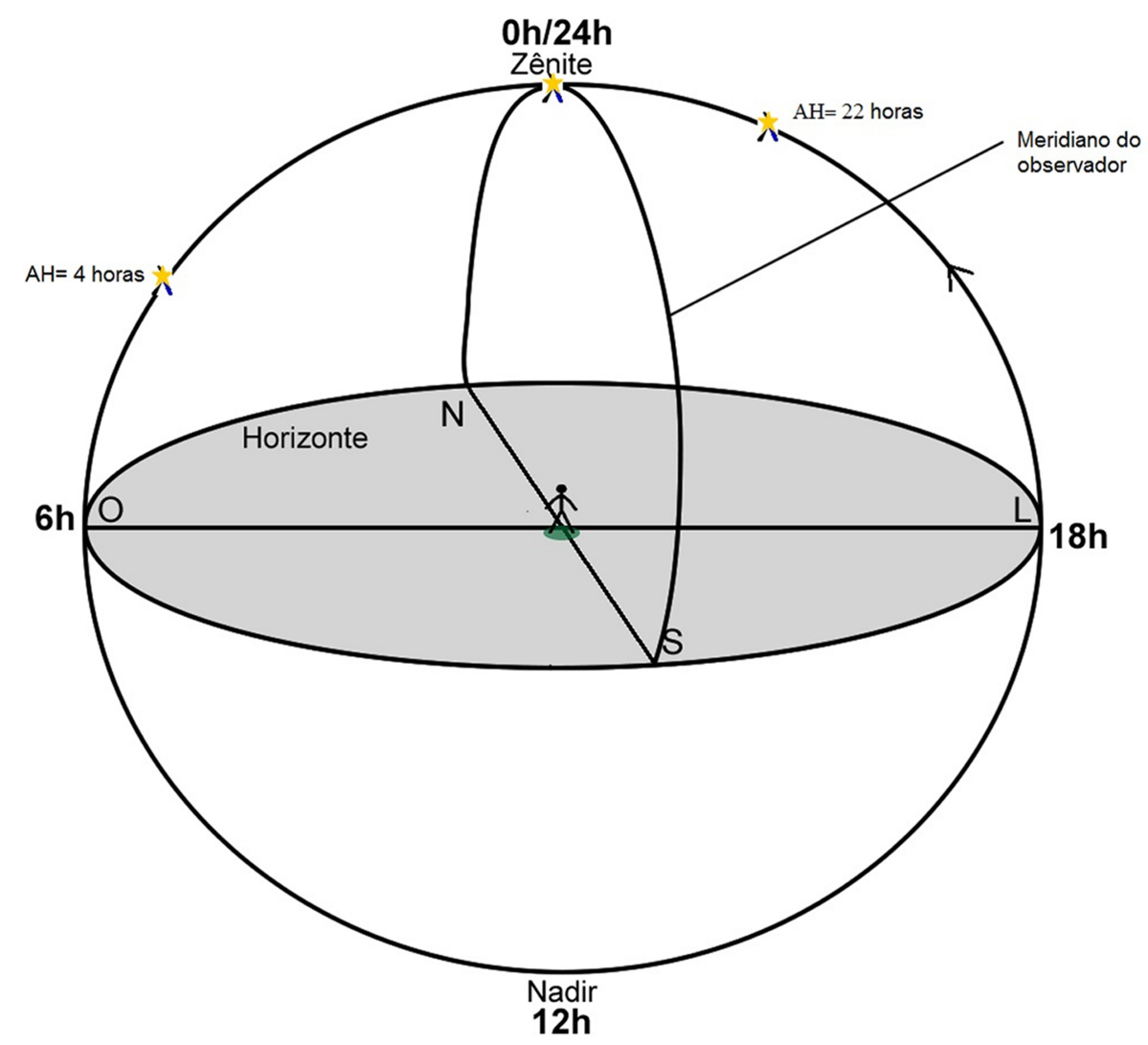

Figura 5: $\mathrm{O}$ ângulo horário $(\mathrm{AH})$. A figura mostra uma estrela com três $\mathrm{AH}$ diferentes ao longo de uma noite. Antes, no momento e após ela cruzar o meridiano do observador.

$21^{0} 34^{\prime} 52$ " Sul e longitude $=46^{0} 50$ '55" Oeste. Para construí-la, primeiro nós utilizamos a Eq. (1) para calcular o dia juliano, JD $=2457546.45833$. Em seguida, utilizamos as equações (4), (5)e(6) para calcular o TSG e, com essa informação, calcular o Tempo Sideral Local (Eq.3), TSL $=13 \mathrm{~h} 2 \mathrm{~m} 23 \mathrm{~s}$. Conhecendo o TSL utilizamos a equação (7) para calcular o AH de cada uma das estrelas da tabela e selecionamos àquelas que estão acima do horizonte. Por último, com as equações (8a) e (8b) calculamos as coordenadas horizontais das estrelas e construímos a carta celeste. Na carta da Fig. 6 apresentamos as estrelas mais brilhantes de algumas constelações que estão visíveis.

Na Tabela 1 estão, a título de comparação, as coordenadas horizontais calculadas nesse trabalho e as coordenadas horizontais obtidas do software cartes du ciel [7] para 3 estrelas da carta celeste. Podemos observar que os valores de $\mathrm{Az}$ e $\mathrm{h}$ obtidos nesse trabalho são compatíveis com os software, a diferença é menor do que 1 grau, tanto para o azimute quanto para a altura. Isso reforça nosso argumento de que com esse método simplificado, que não leva em conta as correções periódicas das coordenadas, é possível construir uma carta celeste.

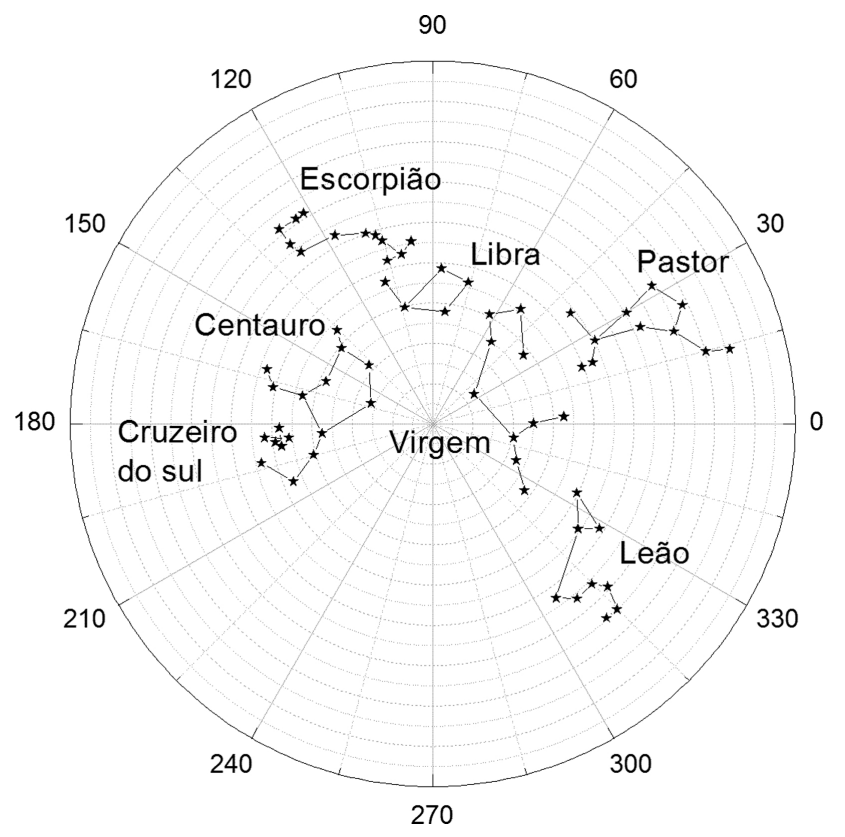

Figura 6: Carta celeste construída com o método desenvolvido nesse trabalho. Nela estão algumas das constelações visíveis no dia $6 / 6 / 2016$ às $20: 00$ horas na latitude $=$ $21^{0} 34^{\prime} 52$ " Sul e longitude $=46^{0} 50^{\prime} 55^{\prime \prime}$ Oeste. 
Tabela 1: Comparação entre as coordenadas horizontais calculadas nesse trabalho com as do software cartes du ciel.

\begin{tabular}{lccc}
\hline Estrela & $\mathrm{Az} \backslash \mathrm{h}$ (neste trabalho) & $\mathrm{Az} \backslash \mathrm{h}$ (literatura) & $|\Delta \mathrm{Az}| \backslash|\Delta \mathrm{h}|$ \\
\hline Alp Sco & 106,8752 & 106,9176 & 0,0424 \\
& 41,1693 & 41,0905 & 0,0788 \\
\hline Gam Cru & 185,5683 & 185,4531 & 0,1152 \\
& 54,1754 & 54,1218 & 0,0536 \\
\hline Bet Leo & 334,4100 & 334,4583 & 0,0483 \\
& 50,3952 & 50,5004 & 0,1051 \\
\hline
\end{tabular}

Nesse trabalho nós não incluímos os astros do sistema solar. Isso porque, diferente das estrelas que estão muito longe e por isso as suas coordenadas equatoriais variam muito lentamente, o sol, a lua e os planetas estão próximos, e, neste caso, não possuem coordenadas equatoriais fixas. Para eles, a cada momento da observação essas coordenadas precisam ser recalculas por um procedimento que está além do escopo desse trabalho. Os leitores que desejarem incluir os astros do sistema solar na carta celeste podem utilizar as Refs $[8,9]$.

\section{Considerações finais}

A proposta apresentada nesse trabalho para construir a carta celeste é utilizada na disciplina de fundamentos de astronomia do curso de licenciatura em física e na disciplina física contemporânea do programa de pós-graduação do mestrado nacional profissional em ensino de física.

Ela também já foi testada com sucesso com alunos do terceiro ano do ensino médio de uma escola pública participante do PIBID. Como quase sempre acontece quando os alunos têm a oportunidade de participar de uma atividade diferente das tradicionais em uma aula de física, a resposta foi gratificante para o professor. Neste caso o professor, com a colaboração dos bolsista do PIBID, utilizou o argumento de construir a carta celeste para introduzir nas aulas de física alguns conteúdos de astronomia, tais como: o movimento dos astros, a contagem do tempo e os sistemas de coordenadas celestes, que dificilmente chamariam a atenção dos estudantes se não houvesse uma motivação.

Em condições regulares essa é uma atividade que pode ser desenvolvida em três aulas de 50 minutos. $\mathrm{Na}$ primeira são trabalhos os conceitos de tempo e sistemas de coordenadas, na segunda são feitos os cálculos das coordenadas horizontais para uma estrela e na terceira a construção da carta celeste com uma planilha de cálculo.

A construção da carta celeste pelo método apresentado neste artigo parece ser uma boa proposta tanto para ensinar conceitos astronômicos como conteúdos da disciplina de matemática no laboratório de informática. Isso foi observado tanto pelos autores do artigo quanto os professores de física e matemática que participaram das atividades na escola quanto os que cursaram a disciplina de fundamentos de astronomia na pós-graduação e já desenvolveram essa atividade na sala de aula.

\section{Apêndice}

\begin{tabular}{lll}
\hline Const & DEC & RA \\
\hline ZetVir & -0.59594 & 13.57827 \\
\hline TauVir & 1.54458 & 14.02744 \\
\hline 109Vir & 1.89294 & 14.77083 \\
\hline MuVir & -5.65965 & 14.7177 \\
\hline IotVir & -5.99953 & 14.26691 \\
\hline AlpVir & -11.16124 & 13.41989 \\
\hline GamVir & -1.44952 & 12.69445 \\
\hline DelVir & 3.3976 & 12.9268 \\
\hline EpsVir & 10.9591 & 13.03632 \\
\hline EtaVir & -0.66675 & 12.33178 \\
\hline
\end{tabular}




\begin{tabular}{|c|c|c|}
\hline BetVir & 1.76538 & 11.8448 \\
\hline EpsLeo & 23.77428 & 9.7642 \\
\hline ZetLeo & 23.41733 & 10.27817 \\
\hline EtaLeo & 16.76267 & 10.12221 \\
\hline AlpLeo & 11.9672 & 10.13957 \\
\hline TheLeo & 15.42976 & 11.23734 \\
\hline BetLeo & 14.57234 & 11.81774 \\
\hline DelLeo & 20.52403 & 11.23511 \\
\hline IotCnc & 28.76517 & 8.77778 \\
\hline GamCnc & 21.4686 & 8.72145 \\
\hline DelCnc & 18.15486 & 8.74475 \\
\hline AlpCnc & 11.85777 & 8.97478 \\
\hline BetCnc & 9.18566 & 8.27526 \\
\hline KsiGem & 12.89606 & 6.75484 \\
\hline GamGem & 16.39941 & 6.62853 \\
\hline ZetGem & 20.5703 & 7.06848 \\
\hline DelGem & 21.98234 & 7.33539 \\
\hline BetGem & 28.02631 & 7.75538 \\
\hline AlpGem & 31.88864 & 7.57667 \\
\hline EpsGem & 25.13116 & 6.7322 \\
\hline MuGem & 22.51309 & 6.38269 \\
\hline EtaGem & 22.50682 & 6.24797 \\
\hline BetTau & 28.60787 & 5.43819 \\
\hline TauTau & 22.95698 & 4.70408 \\
\hline EpsTau & 19.18052 & 4.47693 \\
\hline DelTau & 17.54258 & 4.38223 \\
\hline GamTau & 15.6277 & 4.32987 \\
\hline AlpTau & 16.50976 & 4.59867 \\
\hline ZetTau & 21.14259 & 5.62741 \\
\hline LamTau & 12.49038 & 4.01134 \\
\hline KsiTau & 9.73277 & 3.45281 \\
\hline DelAri & 19.7267 & 3.1938 \\
\hline AlpAri & 23.46278 & 2.11952 \\
\hline BetAri & 20.8083 & 1.91065 \\
\hline IotAri & 17.81759 & 1.95584 \\
\hline TauPsc & 30.08973 & 1.19433 \\
\hline UpsPsc & 27.26409 & 1.32444 \\
\hline PhiPsc & 24.58376 & 1.22915 \\
\hline EtaPsc & 15.34583 & 1.52472 \\
\hline OmiPsc & 9.15764 & 1.75655 \\
\hline AlpPsc & 2.76376 & 2.03411 \\
\hline NuPsc & 5.48762 & 1.69052 \\
\hline MuPsc & 6.14361 & 1.50318 \\
\hline EpsPsc & 7.89007 & 1.04907 \\
\hline OmePsc & 6.86359 & 23.9885 \\
\hline IotPsc & 5.62735 & 23.66578 \\
\hline ThePsc & 6.3791 & 23.46616 \\
\hline GamPsc & 3.28225 & 23.28597 \\
\hline
\end{tabular}




\begin{tabular}{|c|c|c|}
\hline BetPsc & 3.82007 & 23.06461 \\
\hline LamPsc & 1.78042 & 23.7008 \\
\hline EpsAqr & -9.49569 & 20.79459 \\
\hline BetAqr & -5.57116 & 21.52598 \\
\hline OmiAqr & -2.15534 & 22.05523 \\
\hline GamAqr & -1.38735 & 22.36092 \\
\hline LamAqr & -7.57968 & 22.87691 \\
\hline DelAqr & -15.82076 & 22.91084 \\
\hline PhiAqr & -6.04853 & 23.2387 \\
\hline DelCap & -16.12657 & 21.78397 \\
\hline EpsCap & -19.46601 & 21.61801 \\
\hline ZetCap & -22.41138 & 21.44445 \\
\hline OmeCap & -26.91913 & 20.86369 \\
\hline PsiCap & -25.27052 & 20.76827 \\
\hline RhoCap & -17.81367 & 20.48101 \\
\hline BetCap & -14.7814 & 20.35018 \\
\hline TheCap & -17.23271 & 21.09911 \\
\hline IotCap & -16.83456 & 21.37077 \\
\hline GamCap & -16.66225 & 21.66815 \\
\hline AlpSgr & -40.61565 & 19.3981 \\
\hline ZetSgr & -29.88011 & 19.04353 \\
\hline EpsSgr & -34.38431 & 18.40287 \\
\hline GamSgr & -30.42365 & 18.09681 \\
\hline DelSgr & -29.82804 & 18.34989 \\
\hline LamSgr & -25.42125 & 18.46619 \\
\hline PhiSgr & -26.99078 & 18.76093 \\
\hline SigSgr & -26.29659 & 18.92109 \\
\hline TauSgr & -27.66981 & 19.11568 \\
\hline ZetSgr & -29.88011 & 19.04353 \\
\hline LamSco & -37.10375 & 17.56015 \\
\hline KapSco & -39.02992 & 17.56015 \\
\hline TheSco & -42.99782 & 17.62198 \\
\hline EtaSco & -43.23849 & 17.20255 \\
\hline EpsSco & -34.29261 & 16.83618 \\
\hline TauSco & -28.21596 & 16.59804 \\
\hline Alp Sco & -26.43195 & 16.49013 \\
\hline Sig Sco & -25.59275 & 16.35315 \\
\hline Del Sco & -22.62162 & 16.00556 \\
\hline Pi. Sco & -26.11423 & 15.98086 \\
\hline Rho Sco & -29.21401 & 15.94808 \\
\hline SigLib & -25.28186 & 15.06785 \\
\hline BetLib & -9.38287 & 15.28346 \\
\hline GamLib & -14.78955 & 15.59209 \\
\hline UpsLib & -28.13507 & 15.61707 \\
\hline EpsCru & -60.40137 & 12.35606 \\
\hline DelCru & -58.7489 & 12.25243 \\
\hline GamCru & -57.11257 & 12.51942 \\
\hline BetCru & -59.68873 & 12.79537 \\
\hline
\end{tabular}




\begin{tabular}{|c|c|c|}
\hline NuOct & -77.39112 & 21.69138 \\
\hline BetOct & -81.38162 & 22.7677 \\
\hline DelOct & -83.66785 & 14.44882 \\
\hline AlpCen & -60.83947 & 14.66094 \\
\hline BetCen & -60.37298 & 14.06373 \\
\hline EpsCen & -53.46636 & 13.6648 \\
\hline ZetCen & -47.28827 & 13.92568 \\
\hline EtaCen & -42.15775 & 14.59179 \\
\hline TheCen & -36.3687 & 14.11148 \\
\hline IotCen & -36.71208 & 13.34335 \\
\hline GamCen & -48.95989 & 12.692 \\
\hline DelCen & -50.72241 & 12.13932 \\
\hline PiCen & -54.49103 & 11.35009 \\
\hline LamCen & -63.01982 & 11.59637 \\
\hline Alp Boo & 19.18727 & 14.26121 \\
\hline Gam Boo & 38.30788 & 14.53465 \\
\hline Lam Boo & 46.08792 & 14.2731 \\
\hline The Boo & 51.85171 & 14.42001 \\
\hline Bet Boo & 40.39064 & 15.03244 \\
\hline Del Boo & 33.3151 & 15.25836 \\
\hline Eps Boo & 27.07417 & 14.74979 \\
\hline Zet Boo & 13.72833 & 14.68581 \\
\hline Eta Oph & -15.72515 & 17.17296 \\
\hline Zet Oph & -10.56715 & 16.61931 \\
\hline DelOph & -3.69398 & 16.2391 \\
\hline LamOph & 1.9841 & 16.51523 \\
\hline KapOph & 9.37506 & 16.96119 \\
\hline AlpOph & 12.56058 & 17.58222 \\
\hline BetOph & 4.56692 & 17.72455 \\
\hline GamOph & 2.70746 & 17.79822 \\
\hline GamCMa & -15.63326 & 7.06264 \\
\hline AlpCMa & -16.71314 & 6.75257 \\
\hline BetCMa & -17.95592 & 6.37833 \\
\hline PiCMa & -20.13632 & 6.92708 \\
\hline DelCMa & -26.39321 & 7.13986 \\
\hline EpsCMa & -28.97209 & 6.9771 \\
\hline ZetCMa & -30.06338 & 6.33855 \\
\hline EtaCMa & -29.30312 & 7.40158 \\
\hline KapOri & -9.6696 & 5.79594 \\
\hline BetOri & -8.20164 & 5.2423 \\
\hline DelOri & -0.29909 & 5.53344 \\
\hline EpsOri & -1.20192 & 5.60356 \\
\hline ZetOri & -1.94258 & 5.67931 \\
\hline AlpOri & 7.40704 & 5.91952 \\
\hline GamOri & 6.34973 & 5.41885 \\
\hline MuOri & 9.64713 & 6.03973 \\
\hline KsiOri & 14.20881 & 6.199 \\
\hline NuOri & 14.76838 & 6.1262 \\
\hline
\end{tabular}




\begin{tabular}{lll}
\hline GamPer & 53.50645 & 3.07994 \\
\hline AlpPer & 49.86124 & 3.40537 \\
\hline DelPer & 47.78765 & 3.71541 \\
\hline NuPer & 42.57857 & 3.75322 \\
\hline EpsPer & 40.01027 & 3.96423 \\
\hline KsiPer & 35.79103 & 3.98275 \\
\hline ZetPer & 31.88366 & 3.9022 \\
\hline OmiPer & 32.28827 & 3.73865 \\
\hline LamPer & 50.35135 & 4.10974 \\
\hline IotPer & 49.6135 & 3.1508 \\
\hline KapPer & 44.85789 & 3.15823 \\
\hline BetPer & 40.95565 & 3.13615 \\
\hline RhoPer & 38.84053 & 3.08625 \\
\hline DelVel & -54.70857 & 8.74505 \\
\hline LamVel & -43.43262 & 9.13327 \\
\hline KapVel & -55.01069 & 9.36856 \\
\hline PsiVel & -40.46689 & 9.5117 \\
\hline MuHya & -16.83665 & 9.5117 \\
\hline LamHya & -12.35444 & 10.1764 \\
\hline AlpHya & -8.65845 & 9.45979 \\
\hline IotHya & -1.14309 & 9.66428 \\
\hline TheHya & 2.31288 & 9.23945 \\
\hline ZetHya & 5.94547 & 8.92321 \\
\hline EtaHya & 3.39866 & 8.7204 \\
\hline SigHya & 3.34136 & 8.64595 \\
\hline DelHya & 5.70376 & 8.62758 \\
\hline EpsHya & 6.41854 & 8.77953 \\
\hline & & \\
\hline & & \\
\hline
\end{tabular}

\section{Referências}

[1] D. Whitehouse, Oldest Star Chart Found, disponível em http://news.bbc.co.uk/2/hi/science/ nature/2679675.stm, acesso em 1/6/2016.

[2] A.F. Herlihy, Renaissance Star Charts, disponível em http://www.press.uchicago.edu/books/ HOC/HOC_V3_Pt1/HOC_VOLUME3_Part1_chapter4. pdf, acesso em 1/6/2016.

[3] K.S. Oliveira e M.F.O. Saraiva, Astronomia e Astrofísica (Editora Livraria da Física, São Paulo, 2014), v. 1, $3^{\text {a }}$ ed., 780 p.

[4] J.L Lawrence, Basic Astronomy with a PC (Willmann-Bell, Richmond, 1989), v. 1, 1를 ed., 130 p.
[5] G.L.N. Bierrenbach, Astronomia de Posição, disponível em http://www.astro.iag.usp.br/ gastao/AstroPosicao/Curso2016.pdf acesso em $1 / 6 / 2016$.

[6] Documentação disponível em http://www astro.wisc.edu/ dolan/constellations/ constellation_list.html, acesso em 1/6/2016.

[7] Documentação disponível em http://www.ap-i net/skychart/fr/start, acesso em 1/6/2016.

[8] M. Franza and D. Harperb, Planetary and Space Science 50, 217 (2002).

[9] T. Singal and K. Ashok, Determining Planetary Positions in the Sky for \pm 50 Years to an Accuracy of $1^{\circ}$ with a Calculator. Disponível em http://astro.if ufrgs.br/coord/coordplanetas.pdf, acesso em $1 / 6 / 2016$. 